\title{
PLANNING OF COLLISION-FREE TRAJECTORY FOR MOBILE MANIPULATORS
}

\author{
G. PAJĄK \\ University of Zielona Góra \\ Institute of Computer Science and Production Management \\ Licealna 9, 65-417 Zielona Góra, POLAND \\ E-mail: g.pajak@iizp.uz.zgora.pl
}

\begin{abstract}
A method of planning sub-optimal trajectory for a mobile manipulator working in the environment including obstacles is presented. The path of the end-effector is defined as a curve that can be parameterized by any scaling parameter, the reference trajectory of a mobile platform is not needed. Constraints connected with the existence of mechanical limits for a given manipulator configuration, collision avoidance conditions and control constraints are considered. The motion of the mobile manipulator is planned in order to maximize the manipulability measure, thus to avoid manipulator singularities. The method is based on a penalty function approach and a redundancy resolution at the acceleration level. A computer example involving a mobile manipulator consisting of a nonholonomic platform and a SCARA type holonomic manipulator operating in a two-dimensional task space is also presented.
\end{abstract}

Key words: mobile manipulator, path following, trajectory planning, penalty function, control constraints.

\section{Introduction}

The main task accomplished by the manipulator of a robot is to move the end-effector from a given initial position to a given final position in the workspace. In order to extend performance capabilities of the manipulator, its arm is mounted on a mobile wheeled platform. A robotic system consisting of a mobile platform and a manipulator mounted on top is called a mobile manipulator. By combining the mobility of the platform with the dexterous capability of the manipulator, such a system gains kinematic redundancy. The redundant degrees of freedom render it possible to accomplish complex tasks in complicated workspaces with obstacles.

This paper presents a motion planning method for applications, where only the knowledge of the end-effector path is needed (handling and stacking operations, parts assembly or inspection missions). The path of the end-effector is a curve that can be parameterised by any scaling parameter (except for the time parameter) e.g. the length of the path. The robot's trajectory is planned in a manner to maximise the manipulability measure to avoid manipulator singularities. Nonholonomic constraints are incorporated explicitly to the control algorithm, and in this way the algorithm guarantees finding continuous controls which do not exceed their limits. It also ensures the fulfilment of the constraints imposed by the robot's mechanical limits and collision avoidance conditions.

In the proposed solution the dynamic model of the mobile robot is derived from Lagrange's form of D'Alembert's principle. In addition, it is assumed that kinematic and dynamic parameters are fully known, even if they cannot be measured directly they can be estimated by an identification technique (An et al. (1988) and Renders et al. (1991)). Finally, the path following problem is transformed here into an optimisation problem with holonomic and nonholonomic equality constrains, and inequality constraints resulting from mechanical limitations and collision avoidance conditions. The resulting trajectory is scaled in a manner to fulfil the constraints imposed on the controls. The task was solved by using interior penalty 
functions and a redundancy resolution at the acceleration level. The property of asymptotic stability of the proposed solution implies fulfilment of all the constraints imposed.

To the best of the author's knowledge, no research has considered the problem formulated in the above manner. Much of the existing research addresses the problem using only kinematic equations of the mobile manipulator, and the dynamics of the robot is not considered at all. All works known to the author, which take into account the kinematic and dynamic model, require transformation of the nonholonomic constraints in a Pfaffian form to a driftless control system (Galicki, 2011a; 2011b; 2012; Mazur, 2007 and 2010). This transformation is not unique which makes difficult to choose a suitable driftless dynamic system. Opposite to these approaches, presented method incorporates nonholonomic constraints in a Pfaffian form explicitly to the control algorithm. In Mazur (2007) and Mazur (2010) transformation of nonholonomic constraints leads to a non-optimal solution because of the need to introduce additional task constraints. In this approach optimality is inherently built in to the proposed solution of the robot's task.

The presented solution is distinguished by the method of determining the controls which fulfil the assumed constraints. Moreover, proposed method, using the known scaling techniques (Pająk and Pająk, 1997; Pająk and Galicki, 1999), produces the mobile manipulator trajectory parameterised by gain coefficients in such away as to make the corresponding controls fulfil the imposed constraints. Furthermore, the obtained controls are continuous and they can preserve a certain safety margin due to mobile manipulator dynamic equation modelling uncertainty. Other research considering the dynamics of the robot (Galicki, 2011a; 2011b; 2012; Mazur, 2007; 2010) produces controls parameterised by gain coefficients of the controller directly. In such cases, it is very difficult (or impossible) to find coefficients which fulfil control limits.

Moreover, presented solution maximises the manipulability measure of whole manipulator. In consequence, a robot is far from its singular configurations during execution the task (much of existing literature does not deal with any optimality at all). This property ensures a high dexterity of the robot and decreases the probability of the need for the time consuming reconfiguration process. Opposite to similar research, the proposed solution does not require the reference path for the platform, which makes it possible to apply this method in complicated workspaces including many obstacles.

The paper is organised as follows. Section 2 formulates the problem of optimal control. A solution to this problem is presented in Section 3. The proposed method is demonstrated numerically in Section 4, for a mobile manipulator consisting of a nonholonomic $(2,0)$ class platform and a SCARA type holonomic manipulator operating in a two-dimensional task space.

\section{Problem formulation}

A mobile manipulator composed of a nonholonomic platform and holonomic manipulator with kinematic pairs of the $5^{\text {th }}$ class (rotational and translational) is considered to plan a trajectory. It is described by the vector of generalised coordinates

$$
\boldsymbol{q}=\left(\begin{array}{l}
\boldsymbol{q}^{p} \\
\boldsymbol{q}^{r}
\end{array}\right)
$$

where: $\boldsymbol{q} \in \mathfrak{R}^{n}-$ the vector of the generalised coordinates of the whole mobile manipulator; $\boldsymbol{q}^{p} \in \mathfrak{R}^{p}-$ the vector of the coordinates of the nonholonomic platform; $\boldsymbol{q}^{r} \in \mathfrak{R}^{r}$ - the vector of joints coordinates of the holonomic manipulator; $p$-the number of coordinates describing the nonholonomic platform; $r-$ the number of kinematic pairs of the holonomic manipulator; $n=p+r$.

The platform motion is subject to nonholonomic constraints which can be described in the Pfaffian form 


$$
\tilde{\boldsymbol{A}}\left(\boldsymbol{q}^{p}\right) \dot{\boldsymbol{q}}^{p}=\mathbf{0}
$$

where: $\tilde{\boldsymbol{A}}\left(\boldsymbol{q}^{p}\right)-(k \times p)$ Pfaffian full rank matrix; $k$ - the number of nonholonomic constraints.

The position and orientation of the end-effector is described by the kinematic equations $f(\boldsymbol{q})$, where $\boldsymbol{f}: \mathfrak{R}^{n} \rightarrow \mathfrak{R}^{m}$ denotes $m$-dimensional mapping, nonlinear with respect to $\boldsymbol{q}$ and $n, m$ represent number of the coordinates of whole mobile manipulator and the dimension of the workspace, respectively.

To determine the mobile manipulator motion, it is also necessary to know the robot dynamic equations, given in a general form, as

$$
\boldsymbol{M}(\boldsymbol{q}) \ddot{\boldsymbol{q}}+\boldsymbol{F}(\boldsymbol{q}, \dot{\boldsymbol{q}})+\boldsymbol{A}^{T} \boldsymbol{\lambda}=\boldsymbol{B} \boldsymbol{u}
$$

where: $\boldsymbol{M}(\boldsymbol{q})-(n \times n)$ positive definite inertia matrix, $\boldsymbol{F}=\boldsymbol{F}(\boldsymbol{q}, \dot{\boldsymbol{q}})-n$-dimensional vector representing Coriolis, centrifugal, viscous, Coulomb friction and gravity forces; $\boldsymbol{A}(\boldsymbol{q})=\left[\tilde{\boldsymbol{A}}\left(\boldsymbol{q}^{p}\right) \quad \boldsymbol{0}_{k \times r}\right] ; \boldsymbol{0}_{k \times r}-(k \times r)$ zero matrix; $\lambda$-k-dimensional vector of the Lagrange multipliers corresponding to nonholonomic constraints (2); $\boldsymbol{A}^{T} \lambda$ represents the constraint force which enforce fulfilment of nonholonomic constraints; $\boldsymbol{u}-(n-k)$-dimensional vector of controls (torques / forces); $\boldsymbol{B}-(n \times(n-k))$ full rank matrix (by definition) describing which state variables of the mobile manipulator are directly driven by the actuators. follows

The task of the manipulator is to follow a path given in a parametric form. It can be described as

$$
\boldsymbol{f}(\boldsymbol{q})-\boldsymbol{\varphi}(s)=\boldsymbol{0}
$$

where: $\boldsymbol{q}$ - the vector of generalised coordinates which depends on time $t$, i.e., $\boldsymbol{q}=\boldsymbol{q}(t) ; t \in[0, T] ; T$ stands for an unknown time of performing the task (4); $\varphi(s)$ - the function representing a path of the end-effector; $s$ - the scaling parameter which depends on time $t$, i.e., $s=s(t) ; s \in\left[0, s_{\max }\right]$.

The function $\varphi(s)$ describes the position and/or orientation of the end-effector in the workspace. The form of this function depends on the nature of the robot task. It is determined by a higher level module as a function parameterised by any scaling parameter (except for the time parameter) e.g., the length of the path.

The assumption that the manipulator at the initial moment of motion, i.e., for $t=0$, is in the acceptable configuration is taken into account. Additionally, for this configuration the end-effector of the manipulator should be at the initial location of the path

$$
\boldsymbol{f}\left(\boldsymbol{q}_{0}\right)-\boldsymbol{\varphi}(0)=0
$$

where: $\boldsymbol{q}_{0}=\boldsymbol{q}(0)$ denotes initial configuration of the mobile manipulator.

The practical processes that are accomplished by the industrial robots impose some conditions at the beginning and end of a trajectory. It is natural to assume that at the initial and final moments of the task performance, the velocities of the manipulator equal zero

$$
\dot{\boldsymbol{q}}(0)=\mathbf{0}, \quad \dot{s}(0)=0, \quad \dot{\boldsymbol{q}}(T)=\mathbf{0}, \quad \dot{s}(T)=0 .
$$


Constraints connected with the existence of mechanical limits for the manipulator configuration $\boldsymbol{q}$ and the fact that the manipulator links should not collide with the obstacle will be considered. They have the following general form

$$
\forall t \in[0, T] \quad\left\{\beta_{i}(\boldsymbol{q}(t)) \geq 0\right\}, \quad i=1: L,
$$

where: $\left\{\beta_{i}(\boldsymbol{q}(t)) \geq 0\right\}$ - the set of conditions with scalar functions $\beta_{i}()$ which involve the fulfilment of the constraints imposed by the robot mechanical limits and collision avoidance conditions; $L$ means the total number of inequality constraints.

Additionally, the constant constraints of control are also assumed

$$
\boldsymbol{u}_{\min } \leq \boldsymbol{u} \leq \boldsymbol{u}_{\max }
$$

where: $\boldsymbol{u}_{\min }=\left(u_{\min 1}, \ldots, u_{\min n-k}\right)^{T} ; \boldsymbol{u}_{\max }=\left(u_{\max 1}, \ldots, u_{\max n-k}\right)^{T}$ are lower and upper limits on $\boldsymbol{u}$, respectively. For simplicity of further calculations and without loss of generality the equalities $\left|u_{\min i}\right|=\left|u_{\max i}\right|$, where $i=1: n-k$, are assumed to be satisfied.

In practice, it is important for the configuration of manipulator's joints to be far away from singular configurations. The assumption corresponds to a search for the trajectory for which the instantaneous performance index is minimized (maximising the manipulability measure) at each time instant $t \in[0, T]$ :

$$
\tilde{I}(\boldsymbol{q})=-\operatorname{det}\left(\boldsymbol{j} \boldsymbol{j}^{T}\right)
$$

where: $\boldsymbol{j}=\frac{\partial \boldsymbol{f}(\boldsymbol{q})}{\partial \boldsymbol{q}}-(m \times n)$ dimensional Jacobian matrix of the mobile robot.

Dependencies (2.4)-(2.9) formulate the robotic task as an optimal control problem expressed in rather general terms. The fact that there are inequality constraints imposed on the vector $\boldsymbol{q}$ makes its solution difficult. The next section will present an approach that renders it possible to solve the above optimisation problem.

\section{Solution}

To solve the problem defined by dependencies (2.4)-(2.9), an approximate implementation of inequality constraints (2.7) is accepted in the work. The approach is to use penalty functions (interior or exterior), presented in Fiacco and McCormic (1968), Findeisen et al. (1977), which cause the inequality constraints to be satisfied, but the performance index (9) to be somewhat increased. The approach presented herein is the explication of the trajectory planning method described in Galicki (1998), Pająk and Galicki (1999), Pająk and Galicki (2000), Pająk and Pająk (2009).

These requirements result in the following function to be minimized

$$
I(\boldsymbol{q})=\tilde{I}(\boldsymbol{q})+\sum_{i=0}^{L} \kappa_{i}\left(\beta_{i}(\boldsymbol{q})\right)
$$

where: $\kappa_{i}()$-the penalty function which associates a penalty with a violation of a constraint (see Numerical example section for exemplary form of this function). 
The solution of the task was divided into two parts: the search for an optimal configuration for a given path point $\varphi(s)$ and trajectory planning from the initial path point to the end point.

The task of searching for an optimal trajectory for a given path point can be described as follows

$$
\begin{aligned}
& \boldsymbol{f}(\boldsymbol{q})-\boldsymbol{\varphi}(s)=\mathbf{0}, \\
& \boldsymbol{A}(\boldsymbol{q}) \dot{\boldsymbol{q}}=\boldsymbol{0}, \\
& \min _{\boldsymbol{q}} I(\boldsymbol{q}) .
\end{aligned}
$$

Based on the solution presented in Pająk and Pająk (2010) and using the necessary condition for the minimum of performance index $I(\boldsymbol{q})$ (Galicki, 1992), the task of searching for an optimal configuration $\mathbf{q}$ for the given path point can be described as

$$
\left.\tilde{\boldsymbol{E}}(\boldsymbol{q}, \dot{\boldsymbol{q}}, s)=\left(\begin{array}{c}
\boldsymbol{f}(\boldsymbol{q})-\boldsymbol{\varphi}(s) \\
{\left[\left(\left(\boldsymbol{J}^{R}(\boldsymbol{q})\right)^{-1} \boldsymbol{J}^{F}(\boldsymbol{q})\right)^{T}-\boldsymbol{1}_{n-m-k}\right.} \\
\boldsymbol{A}(\boldsymbol{q}) \dot{\boldsymbol{q}}
\end{array}\right] \boldsymbol{I}_{q}(\boldsymbol{q})\right)=\boldsymbol{0}
$$

where: $\boldsymbol{J}(\boldsymbol{q})=\left[\begin{array}{ll}(\boldsymbol{j}(\boldsymbol{q}))^{T} & (\boldsymbol{A}(\boldsymbol{q}))^{T}\end{array}\right]^{T}-((m+k) \times n)$ dimensional full rank matrix, the full rank of the matrix is force by maximising the manipulability measure (3.1), $(m+k)<n ; \boldsymbol{J}^{R}(\boldsymbol{q})-((m+k) \times(m+k))$ dimensional matrix constructed from $(m+k)$ linear independent columns of $\quad \boldsymbol{J} ; \quad \boldsymbol{J}^{F}(\boldsymbol{q})-$ $((m+k) \times(n-m-k))$ dimensional matrix obtained by excluding $\boldsymbol{J}^{R}$ from $\boldsymbol{J} ; \boldsymbol{1}_{n-m-k}-$ $(n-m-k) \times(n-m-k)$ identity matrix.

Dependency (3.3) presents a system of $n$ nonlinear equations which fully specify the configuration $q$ in a given path point. To find the trajectory of the manipulator $\boldsymbol{q}(t)$ and the path parameterisation $s(t)$ Eq.(3.3) has been extended by an additional element responsible for tracing the entire path $\left(\boldsymbol{E}^{I}, \boldsymbol{E}^{I I}\right.$ has been introduced for simplicity of further calculations)

$$
\boldsymbol{E}(\boldsymbol{q}, \dot{\boldsymbol{q}}, s)=\left(\begin{array}{c}
\tilde{\boldsymbol{E}}(\boldsymbol{q}, \dot{\boldsymbol{q}}, s) \\
s-s_{\max }
\end{array}\right)=\left(\begin{array}{c}
\boldsymbol{E}^{I}(\boldsymbol{q}, s) \\
\boldsymbol{E}^{I I}(\boldsymbol{q}, \dot{\boldsymbol{q}})
\end{array}\right)
$$

where: $\quad \boldsymbol{E}^{I}(\boldsymbol{q}, s)=\left(\left[\left(\left(\boldsymbol{J}^{R}(\boldsymbol{q})\right)^{-1} \boldsymbol{J}^{F}(\boldsymbol{q})\right)^{T}-\boldsymbol{1}_{n-m-k}\right] \boldsymbol{I}_{q}(\boldsymbol{q})\right) ; \quad \boldsymbol{E}^{I I}(\boldsymbol{q}, \dot{\boldsymbol{q}})=\boldsymbol{A}(\boldsymbol{q}) \dot{\boldsymbol{q}}$. 
In order to solve the trajectory planning problem, the following system of differential equations is introduced

$$
\begin{aligned}
& \ddot{\boldsymbol{E}}^{I}+\boldsymbol{\Lambda}_{V}^{I} \dot{\boldsymbol{E}}^{I}+\boldsymbol{\Lambda}_{L}^{I} \boldsymbol{E}^{I}=\mathbf{0}, \\
& \dot{\boldsymbol{E}}^{I I}+\boldsymbol{\Lambda}_{L}^{I I} \boldsymbol{E}^{I I}=\mathbf{0}
\end{aligned}
$$

where: $\Lambda_{V}^{I}=\operatorname{diag}\left(\Lambda_{V, 1}^{I}, \ldots, \Lambda_{V, n-k+1}^{I}\right), \Lambda_{L}^{I}=\operatorname{diag}\left(\Lambda_{L, 1}^{I}, \ldots, \Lambda_{L, n-k+1}^{I}\right)-((n-k+1) \times(n-k+1))$ diagonal matrices with positive coefficients $\Lambda_{V, i}^{I}, \quad \Lambda_{L, i}^{I}$, ensuring the stability of the first equation; $\Lambda_{L}^{I I}=\operatorname{diag}\left(\Lambda_{L, 1}^{I I}, \ldots, \Lambda_{L, k}^{I I}\right)-(k \times k)$ diagonal matrix with positive coefficients $\Lambda_{L, i}^{I I}$, ensuring the stability of the second equation.

This is a system of homogeneous differential equations with constant coefficients. In order to solve these equations $(2 n-k+2)$ consistent dependencies should be given. These dependencies are obtained from the Eq.(3.4) for $t=0$, taking into account the initial conditions (2.5) and (2.6).

The above form of the system of differential equations (3.5) ensures that its solution is asymptotically stable for positive coefficients $\Lambda_{V, i}^{I}, \Lambda_{L, i}^{I}$ and $\Lambda_{L, i}^{I I}$. The property of asymptotic stability implies fulfilment of the following conditions

$$
\dot{\boldsymbol{q}}(T) \rightarrow \boldsymbol{0} ; \quad s(T) \rightarrow s_{\max } ; \quad \dot{s}(T) \rightarrow 0 \quad \text { as } \quad T \rightarrow \infty .
$$

In addition, if the gain coefficients $\Lambda_{V, i}^{I}$ and $\Lambda_{L, i}^{I}$ satisfy the following inequalities

$$
\Lambda_{V, i}^{I}>2 \sqrt{\Lambda_{L, i}^{I}}
$$

then the function $\boldsymbol{E}^{I}(\boldsymbol{q}, s)$ becomes also a strictly monotonic function. For the initial non-singular configuration $\boldsymbol{q}_{0}$ fulfilling mechanical and collision avoidance constraints (i.e. satisfying dependencies from $m+1$ to $n-k$ of $\boldsymbol{E}^{I}$ ), this property forces fulfilment of mechanical constraints, collision avoidance conditions and singularity-free robotic motions along a given path (2.4).

In order to obtain the trajectory of the manipulator $\boldsymbol{q}(t)$ and the path parameterisation $s(t)$ Eqs (3.5), after simple calculations, can be represented as

$$
\begin{aligned}
& \ddot{s}=-\left(\widehat{\Lambda}_{V}^{I} \dot{s}+\widehat{\Lambda}_{L}^{I}\left(s-s_{\max }\right)\right) \\
& \ddot{\boldsymbol{q}}=-\left[\begin{array}{c}
\breve{\boldsymbol{E}}_{q}^{I} \\
\boldsymbol{E}_{\dot{q}}^{I I}
\end{array}\right]^{-1}\left[\begin{array}{c}
\frac{d}{d t}\left(\breve{\boldsymbol{E}}_{q}^{I}\right) \dot{\boldsymbol{q}}+\breve{\boldsymbol{E}}_{s}^{I} \ddot{s}+\frac{d}{d t}\left(\breve{\boldsymbol{E}}_{s}^{I}\right) \dot{s}+\breve{\Lambda}_{V}^{I}\left(\breve{\boldsymbol{E}}_{q}^{I} \dot{\boldsymbol{q}}+\breve{\boldsymbol{E}}_{s}^{I} \dot{s}\right)+\breve{\boldsymbol{\Lambda}}_{L}^{I} \breve{\boldsymbol{E}}^{I} \\
\boldsymbol{E}_{q}^{I I} \dot{\boldsymbol{q}}+\boldsymbol{\Lambda}_{L}^{I I} \boldsymbol{E}^{I I}
\end{array}\right]
\end{aligned}
$$


where: $\breve{\boldsymbol{E}}^{I}-$ the dependency obtained by excluding $\left(s-s_{\max }\right)$ from $\boldsymbol{E}^{I} ; \breve{\boldsymbol{E}}_{q}^{I}=\frac{\partial \breve{\boldsymbol{E}}^{I}(\boldsymbol{q}, s)}{\partial \boldsymbol{q}} ; \breve{\boldsymbol{E}}_{s}^{I}=\frac{\partial \breve{\boldsymbol{E}}^{I}(\boldsymbol{q}, s)}{\partial s}$; $\breve{\Lambda}_{V}^{I}=\operatorname{diag}\left(\Lambda_{V, 1}^{I}, \ldots, \Lambda_{V, n-k}^{I}\right) ; \quad \breve{\boldsymbol{\Lambda}}_{L}^{I}=\operatorname{diag}\left(\Lambda_{L, 1}^{I}, \ldots, \Lambda_{L, n-k}^{I}\right) ; \quad \boldsymbol{E}_{q}^{I I}=\frac{\partial \boldsymbol{E}^{I I}(\boldsymbol{q}, \dot{\boldsymbol{q}})}{\partial \boldsymbol{q}} ; \quad \boldsymbol{E}_{\dot{q}}^{I I}=\frac{\partial \boldsymbol{E}^{I I}(\boldsymbol{q}, \dot{\boldsymbol{q}})}{\partial \dot{\boldsymbol{q}}} ;$ $\widehat{\Lambda}_{V}^{I}=\Lambda_{V, n-k+1}^{I} ; \widehat{\Lambda}_{L}^{I}=\Lambda_{L, n-k+1}^{I}$.

Equations (3.8)-(3.9) specify the system of differential equations of the second order. The trajectory of a mobile manipulator is the solution of this system. To determine values of controls which are required to realise the trajectory it is necessary to transform the dynamic Eq.(2.3). For this purpose the nonholonomic constraints are expressed by an analytic driftless dynamic system

$$
\dot{\boldsymbol{q}}^{p}=\tilde{\boldsymbol{N}}\left(\boldsymbol{q}^{p}\right) v
$$

where: $\tilde{\boldsymbol{N}}\left(\boldsymbol{q}^{p}\right)-(p \times(p-k))$ dimensional matrix; $v-(p-k)$ dimensional vector denotes scaled angular velocities of the platform driving wheels.

Noting that $\tilde{\boldsymbol{A}}\left(\boldsymbol{q}^{p}\right) \tilde{\boldsymbol{N}}\left(\boldsymbol{q}^{p}\right)=\boldsymbol{0}$, the dynamic Eq.(2.3) can be expressed as

$$
\boldsymbol{N}^{T}(\boldsymbol{q}) \boldsymbol{M}(\boldsymbol{q}) \ddot{\boldsymbol{q}}+\boldsymbol{N}^{T}(\boldsymbol{q}) \boldsymbol{F}(\boldsymbol{q}, \dot{\boldsymbol{q}})=\boldsymbol{N}^{T}(\boldsymbol{q}) \boldsymbol{B} \boldsymbol{u}
$$

where: $\quad \boldsymbol{N}(\boldsymbol{q})=\left[\begin{array}{cc}\tilde{\boldsymbol{N}}\left(\boldsymbol{q}^{p}\right) & \boldsymbol{0}_{p \times r} \\ \boldsymbol{0}_{r \times(p-k)} & 1_{r \times r}\end{array}\right]$.

The above equation allows determination of controls for a current trajectory. In order to ensure fulfilment of constraints (2.8) an idea of trajectory scaling, presented in Pająk and Pająk (1997), Pająk and Galicki (1999), is used. In this approach, scaling is accomplished by varying the values of gain coefficients $\widehat{\Lambda}_{V}^{I}, \widehat{\Lambda}_{L}^{I}, \breve{\Lambda}_{V}^{I}, \breve{\Lambda}_{L}^{I}$. Finally, the solution of Eqs (3.8)-(3.9) with suitable parameters $\boldsymbol{\Lambda}(t)$ gives an optimal trajectory satisfying path constraints (2.4), inequality constraints (2.7) and control constraints (2.8) as well as boundary conditions (2.6).

\section{Numerical example}

In the numerical example, a mobile manipulator consisting of a nonholonomic platform of $(2,0)$ class and a holonomic manipulator of two revolute kinematic pairs is considered. The holonomic part is a SCARA type manipulator working in a two-dimensional task space. In order to increase the degree of its redundancy, the orientation of its end-effector is not taken into consideration. A kinematic scheme of the mobile manipulator is shown in Fig.1. 


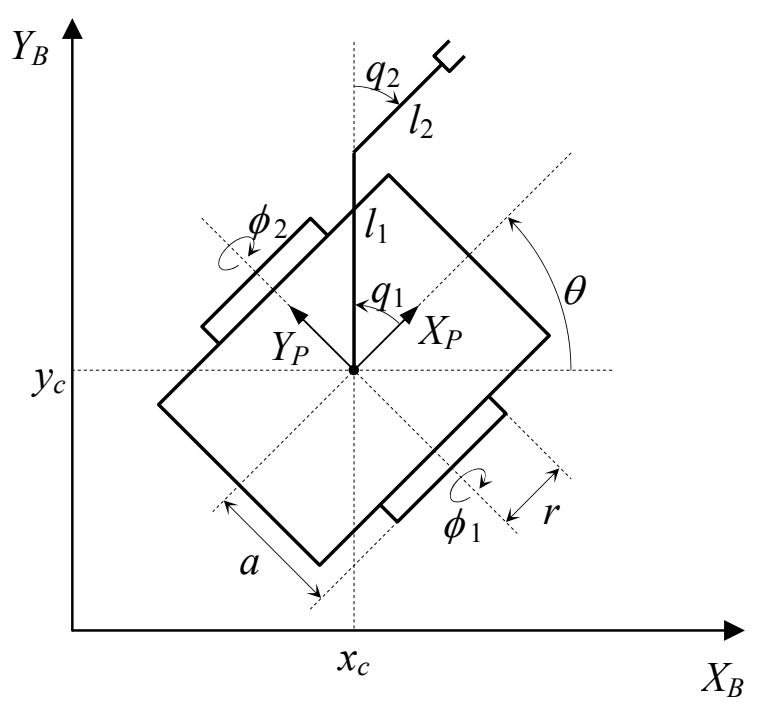

Fig.1. Kinematic scheme of the mobile manipulator.

The platform is described by the vector of generalized coordinates

$$
\boldsymbol{q}^{p}=\left(x_{c}, y_{c}, \theta, \varphi_{1}, \varphi_{2}\right)^{T}
$$

where: $\left(x_{c}, y_{c}\right)$ - the platform center location; $\theta$ - the platform orientation; $\phi_{1}, \phi_{2}$ - angles of driving wheels.

The holonomic manipulator is described by the vector of generalized coordinates

$$
\boldsymbol{q}^{r}=\left(q_{1}, q_{2}\right)^{T}
$$

where: $q_{1}, q_{2}$-joint's angles of the holonomic manipulator.

The mobile manipulator works in $X_{B} Y_{B}$ plane of the base coordinate system $O_{B} X_{B} Y_{B} Z_{B}$. The coordinate system $O_{P} X_{P} Y_{P} Z_{P}$ is attached to the mobile platform at the midpoint of the line segment connecting the two driving-wheels. The holonomic manipulator is connected to the platform at the origin of $O_{P} X_{P} Y_{P} Z_{P}$, the $O_{P} X_{P} Y_{P} Z_{P}$ system can be treated as a base system of the holonomic manipulator. The transformation matrices between the $O_{B} X_{B} Y_{B} Z_{B}$ system, $O_{P} X_{P} Y_{P} Z_{P}$, and the system connected with the last joint of the manipulator $\mathrm{O}_{2} X_{2} Y_{2} Z_{2}$ are given as follows

$$
{ }_{P}^{B} \boldsymbol{T}=\left[\begin{array}{cccc}
\cos (\theta) & -\sin (\theta) & 0 & x_{c} \\
\sin (\theta) & \cos (\theta) & 0 & y_{c} \\
0 & 0 & 1 & 0 \\
0 & 0 & 0 & 1
\end{array}\right], \quad{ }_{2}^{P} \boldsymbol{T}=\left[\begin{array}{cccc}
\cos \left(q_{1}+q_{2}\right) & -\sin \left(q_{1}+q_{2}\right) & 0 & l_{1} \cos \left(q_{1}\right) \\
\sin \left(q_{1}+q_{2}\right) & \cos \left(q_{1}+q_{2}\right) & 0 & l_{1} \sin \left(q_{1}\right) \\
0 & 0 & 1 & 0 \\
0 & 0 & 0 & 1
\end{array}\right]
$$

where: ${ }_{P}^{B} \boldsymbol{T}$ - the position and orientation of the $O_{P} X_{P} Y_{P} Z_{P}$ system in the base system; ${ }_{2}^{P} \boldsymbol{T}$ - the position and orientation of the $\mathrm{O}_{2} X_{2} Y_{2} Z_{2}$ system in the $O_{P} X_{P} Y_{P} Z_{P}$ platform system; $l_{1}$ - the length of the first manipulator arm.

The end-effector is located at the point $\left[l_{2}, 0,0\right]^{T}$ expressed in the $O_{2} X_{2} Y_{2} Z_{2}$ system, where $l_{2}$ is the length of the second arm of manipulator. 
The motion of the platform is subject to one holonomic and two nonholonomic constraints, so constraints (2) in this case take the following form

$$
\left[\begin{array}{ccccc}
0 & 0 & 1 & -\frac{r}{2 a} & \frac{r}{2 a} \\
1 & 0 & 0 & -\frac{r}{2} \cos (\theta) & -\frac{r}{2} \cos (\theta) \\
0 & 1 & 0 & -\frac{r}{2} \sin (\theta) & -\frac{r}{2} \sin (\theta)
\end{array}\right]\left[\begin{array}{c}
\dot{x}_{c} \\
\dot{y}_{c} \\
\dot{\theta} \\
\dot{\varphi}_{1} \\
\dot{\varphi}_{2}
\end{array}\right]=0
$$

where: $r$ - the radius of driving wheels; $a$ - half-distance between the wheels.

The kinematic parameters of the mobile manipulator are given as (all physical values are expressed in the SI system): $l_{1}=l_{2}=0.4, a=0.3, r=0.075$. Respectively, the masses of the mobile manipulator's elements amount to: $m_{p}=104, m_{1}=4.0, m_{2}=4$, where $m_{p}$ is the total mass of the platform and $m_{1}, m_{2}$ are the masses of the manipulator's arm.

The task of the manipulator is to trace a path described by the following equation

$$
\boldsymbol{\varphi}(s)=\left(\begin{array}{c}
2 s-0.6828 \\
s+0.2828
\end{array}\right), \quad s \in[0,2]
$$

Constraints (2.6), (2.8), (2.9) amount to

$$
\begin{aligned}
& \boldsymbol{q}_{0}=(0.0,0.0, \pi / 2,0.0,0.0, \pi / 4, \pi / 4)^{T}, \\
& \boldsymbol{q}_{\max }^{r}=-\boldsymbol{q}_{\min }^{r}=(\pi / 2,3 \pi / 4)^{T}, \\
& \boldsymbol{u}_{\max }=-\boldsymbol{u}_{\min }=(6.0,6.0,1.5,1.0)^{T} .
\end{aligned}
$$

The penalty function introduced in order to take into account mechanical and collision avoidance constraints (2.7) is taken as follows

$$
\kappa_{i}\left(\beta_{i}(\boldsymbol{q})\right)=\left\{\begin{array}{cc}
\rho\left(\beta_{i}(\boldsymbol{q})-\varepsilon_{i}\right)^{2} & \text { for } \quad \beta_{i}(\boldsymbol{q}) \leq \varepsilon_{i} \\
0 & \text { otherwise }
\end{array}\right.
$$

where: $\rho$ - the constant positive coefficient determining the strength of penalty; $\varepsilon_{i}$ - the constant positive coefficient determining the threshold value which activates the $i$-th constraint.

Three cases of performing this task are considered. The first one is an end-effector motion along the path without control constraints (2.8) and collision avoidance constraints. In the second case the mobile manipulator is used to solve the same task as in the first experiment, but control constraints (2.8) are taken into account. In the third simulation, there is additionally an obstacle in the workspace.

In the first case, the values of gain coefficients $\Lambda_{L}, \Lambda_{V}$ are taken as: $\Lambda_{L}^{I}=\operatorname{diag}(1,1,1,1,1)$, $\boldsymbol{\Lambda}_{V}^{I}=\operatorname{diag}(2.1,2.1,2.1,2.1,2.1), \quad \boldsymbol{\Lambda}_{L}^{I I}=\operatorname{diag}(1,1,1)$. The manipulator motion in the work space and corresponding controls obtained in the numerical simulations are shown in Figs 2 and 3. For this solution the final time $T$ is equal to $27.3[\mathrm{~s}$ ], and it can be seen that the determined controls exceed the assumed constraints. 


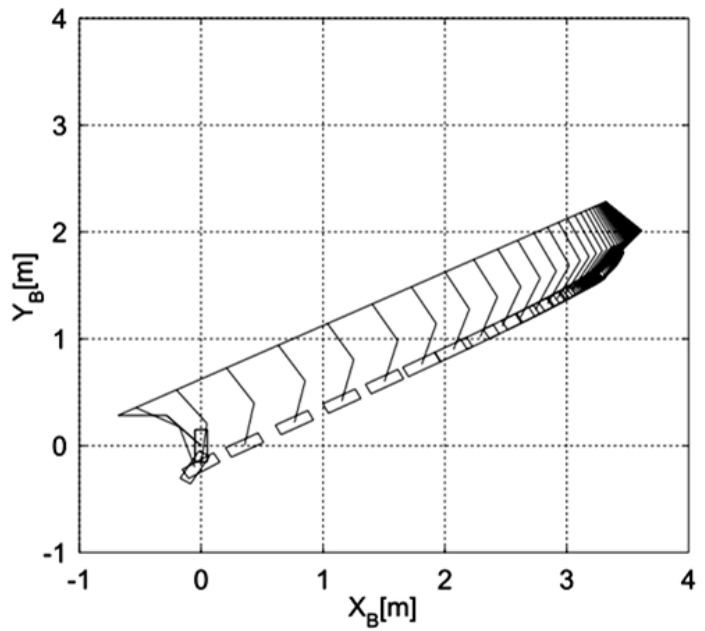

Fig.2. Manipulator motion for the first case.
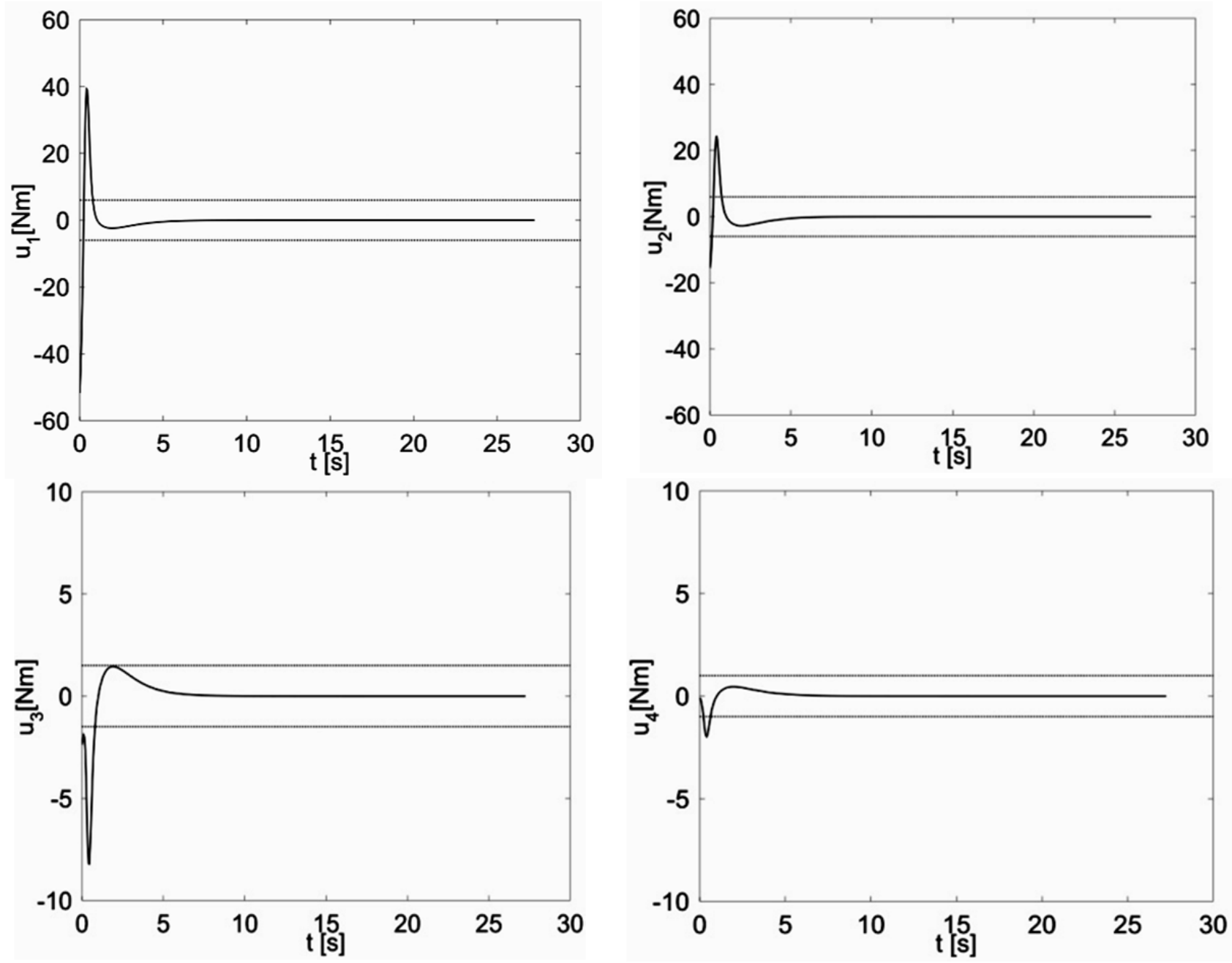

Fig.3. Controls corresponding to the motion for the first case. 
In the second case, control constraints (2.8) are considered. To satisfy these constraints, the values of gain coefficients corresponding to path parameter have been changed and amount to: $\Lambda_{V, 5}^{I}=0.664$, $\Lambda_{L, 5}^{I}=0.1$. For this solution, the final time $T$ is increased and it equals $36.8[s]$, but the determined controls do not exceed the assumed constraints. Figures 4 and 5 present the manipulator motion and controls obtained in this simulations.

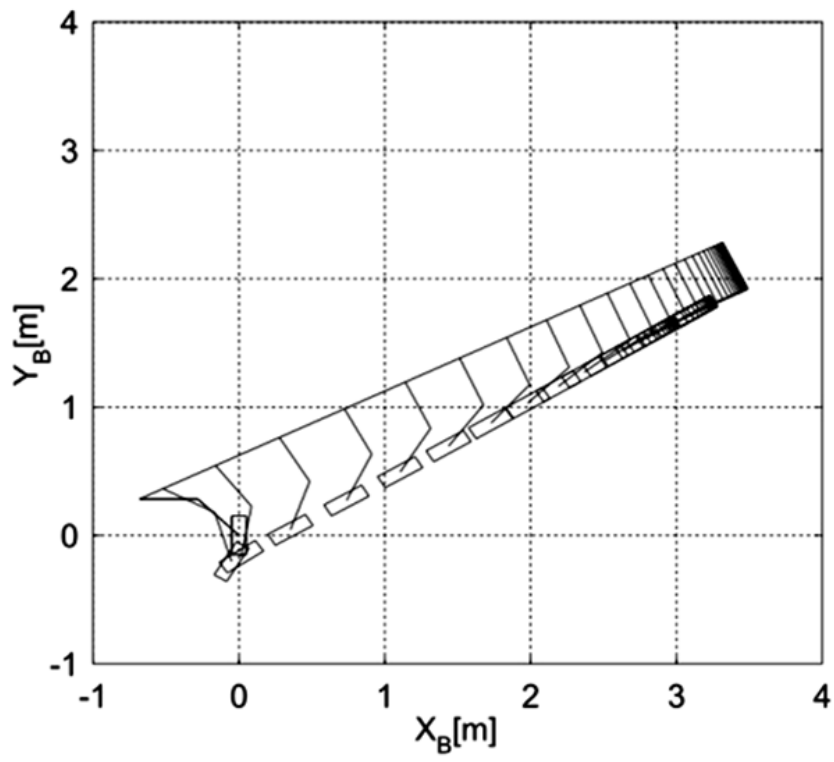

Fig.4. Manipulator motion for the second case.
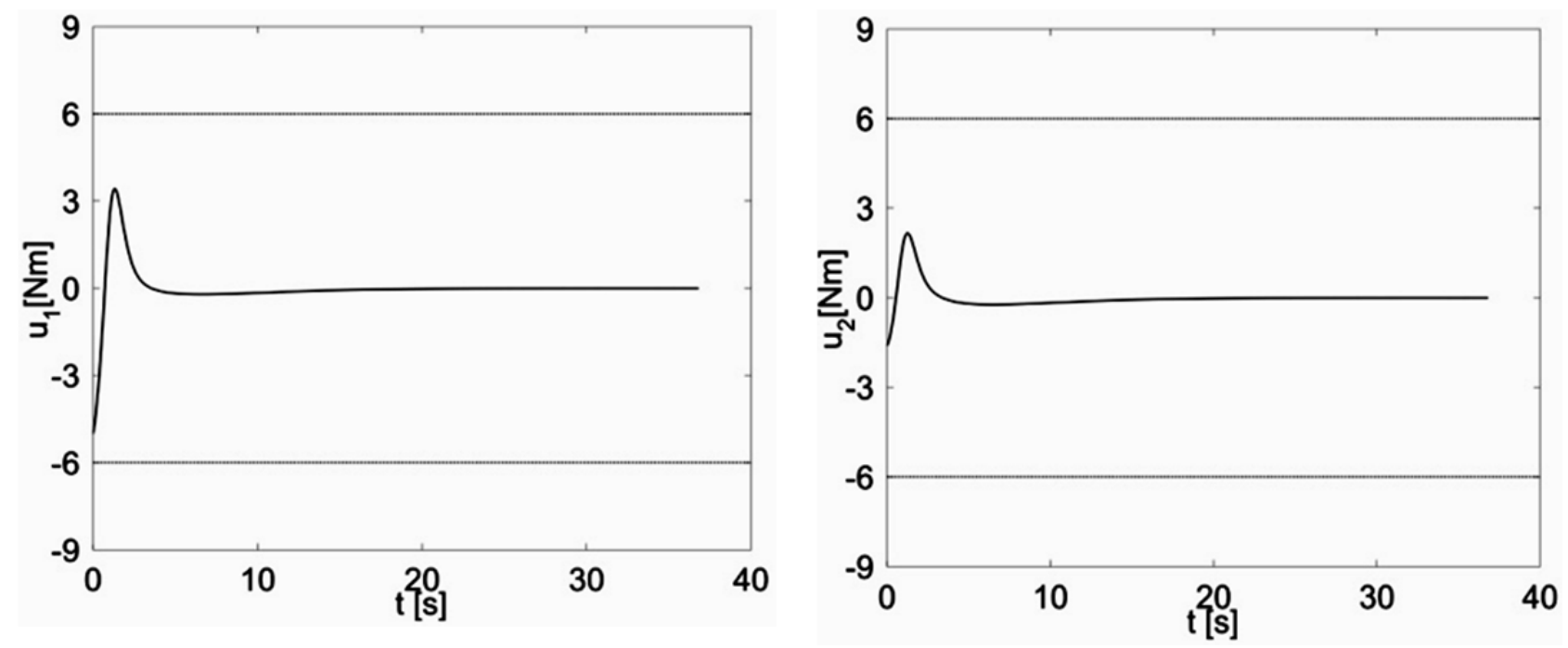

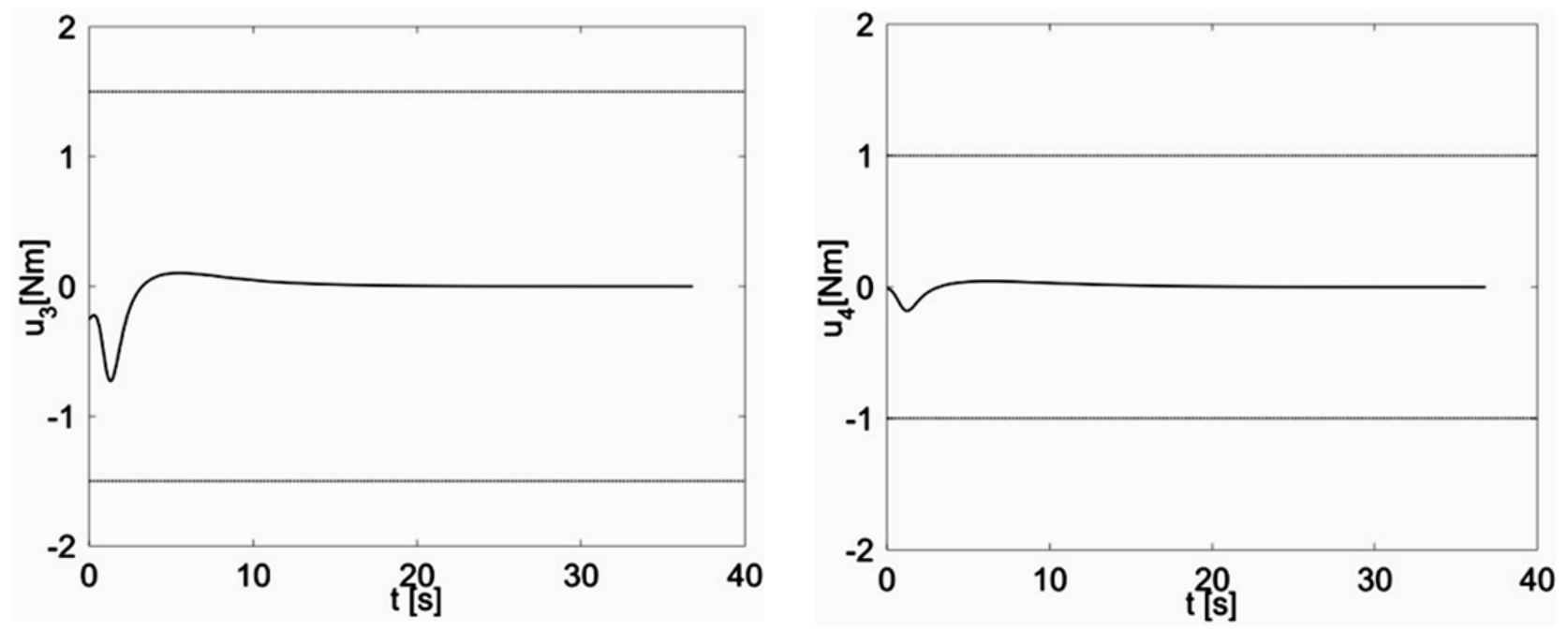

Fig.5. Controls corresponding to the motion for the second case.

In the third simulation, there is an obstacle which may collide with robot. It is approximated by a sphere with center point $(0.5,0.0,0.0)^{T}$ and radius equals 0.25 . Parameters for the simulation are the same as in the previous one. For this solution, the final time $T$ increased even more and it equals $37.7[\mathrm{~s}]$, but both control constraints and collision avoidance constraints are satisfied. Due to the obstacle acting on the mobile manipulator in the initial phase of the motion the controls are slightly sharp. Figures 6 and 7 present the manipulator motion and controls obtained in the simulations.

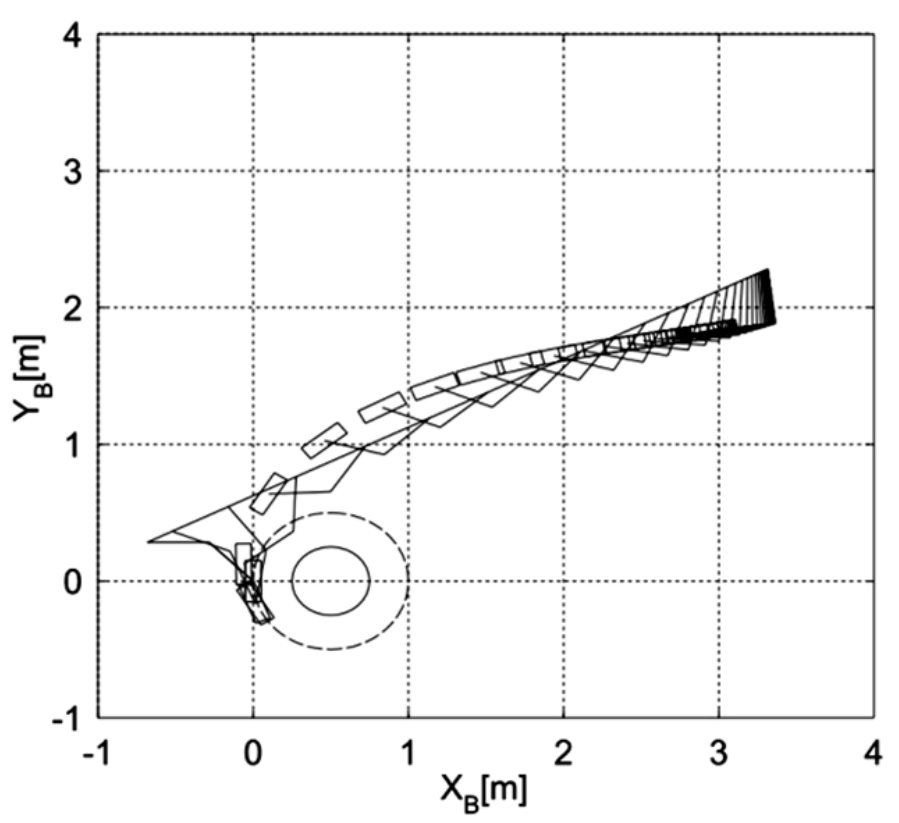

Fig.6. Manipulator motion for the third case. 

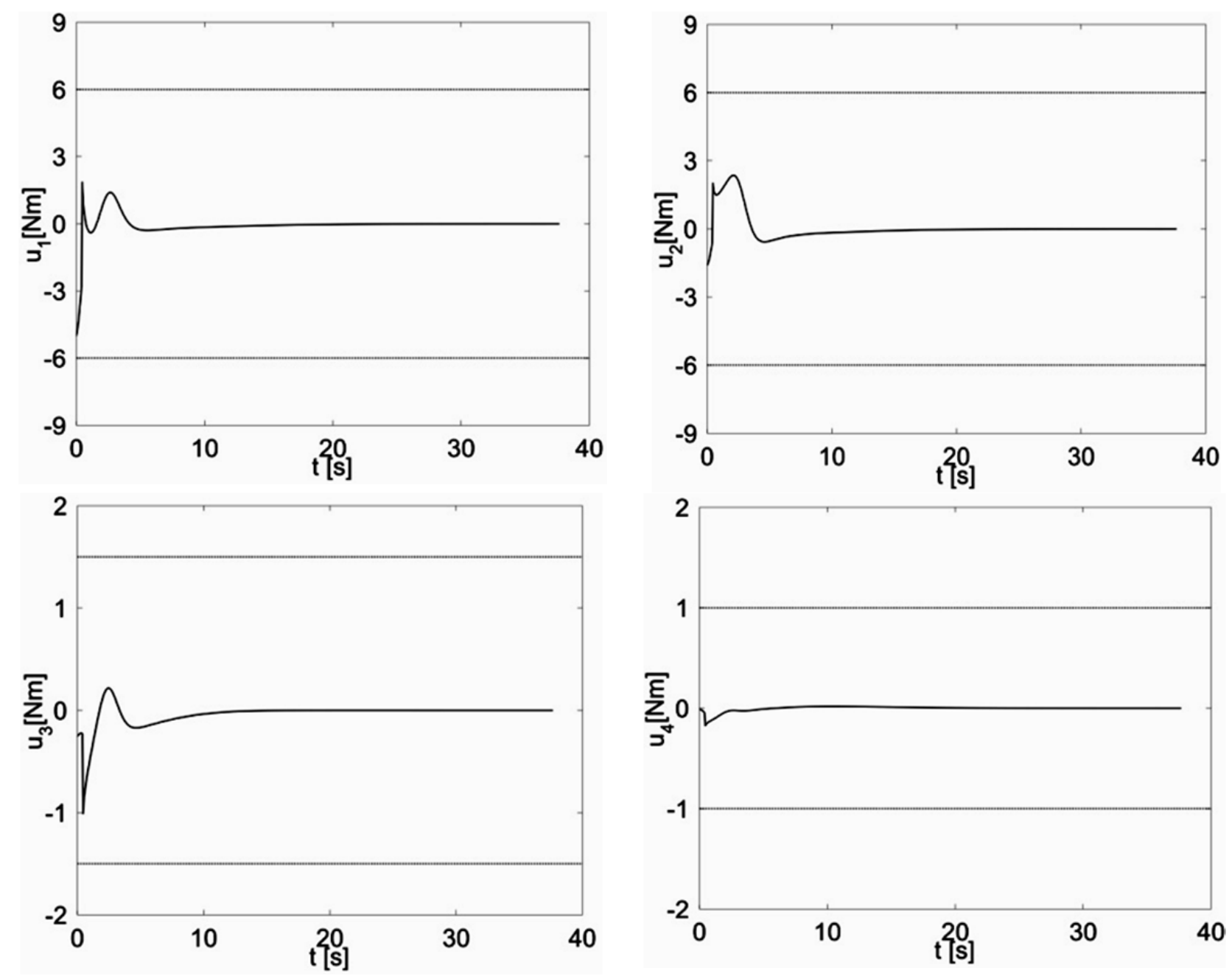

Fig.7. Controls corresponding to the motion for the third case.

\section{Conclusions}

In the paper, a method of trajectory planning for mobile manipulators has been presented. The task of the robot has been to move the tool along the given path that can be parameterised by any scaling parameter. The knowledge of the reference trajectory of a mobile platform has not been needed. The manipulator motion is planned in a manner to maximise the manipulability measure in order to avoid manipulator singularities. Constraints connected with the existence of mechanical limitations for manipulator configuration, collision avoidance conditions and control constraints have been considered. To the best of the author's knowledge, no research has considered the problem formulated in the above manner.

The problem is solved by using penalty functions and a redundancy resolution at the acceleration level. The method presented in the paper ensures finding continuous controls. The proposed approach to trajectory planning is a computationally efficient method. The effectiveness of the solution is confirmed by the results of computer simulations.

\section{Nomenclature}

$\tilde{A}-$ Pfaffian full rank matrix

$\boldsymbol{A}$ - extended Pfaffian matrix 


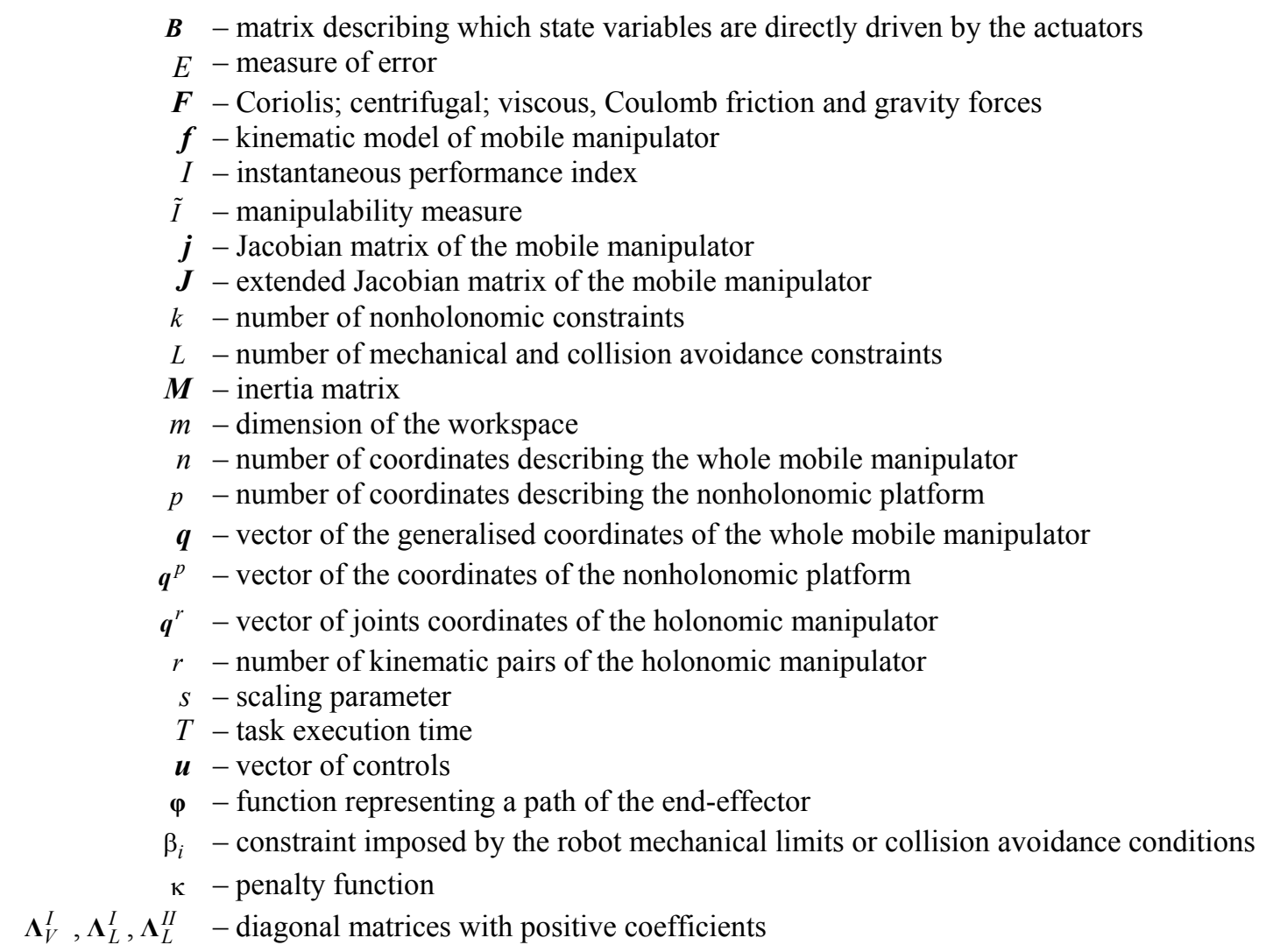

\section{References}

An C.H., Atkeson C.G. and Hollerbach J.M. (1988): Model-based control of a robot manipulator. - Cambridge: Mit Press.

Fiacco A.V. and McCormick G.P. (1968): Nonlinear Programming: Sequential Unconstrained Minimization Techniques. - John Wiley \& Sons, New York.

Findeisen W., Szymanowski J. and Wierzbicki A. (1977): Theory and Methods of Optimization (in Polish). - Polish Scientific Publisher, Warsaw.

Galicki M. (1998): The planning of robotic optimal motions in the presence of obstacles. - Int. J. Rob. Res., vol.17, No.3, pp.248-259.

Galicki M. (2011a): Task space control of mobile manipulators. - Robotica, vol.29, pp.221-232.

Galicki M. (2011b): Collision-free control of mobile manipulators in a task space. - Mech. Syst. Sig. Proc., vol.25, No.7, pp.2766-2784.

Galicki M. (2012): Two-stage constrained control of mobile manipulators. - Mechanism and Machine Theory, vol.54, pp.18-40.

Mazur A. (2007): Path following for nonholonomic mobile manipulators. - Rob. Motion and Control, LNCIS 360 , pp.279-292.

Mazur A. (2010): Trajectory tracking control in workspace-defined tasks for nonholonomic mobile manipulators. Robotica, vol.28, pp.57-68.

Pająk G. and Galicki, M. (2000): Collision-free trajectory planning of the redundant manipulators. - Proc. of the Methods and Models in Autom. and Rob., vol.2, pp.605-610. 
Pająk G. and Pająk I. (2009): Sub-optimal trajectory planning of the redundant manipulators. - Int. J. Appl. Mech. Eng., vol.14, No.1, pp.251-260.

Pająk G. and Pająk I. (1997): Planning of an optimal trajectory of redundant manipulator with regard to constraints on control. - Arch. Mech. Eng., vol.44, No.1, pp.5-20.

Pająk I. and Galicki M. (1999): The planning of suboptimal collision-free robotic motions. - In: Proc. of the Rob. Motion and Control, pp.229-243.

Renders J.M., Rossignol E., Becquet M. and Hanus R. (1991): Kinematic calibration and geometrical parameter identification for robots. - IEEE Trans. Rob. Autom., vol.7, No.6, pp.721-731.

Received: September 23, 2012

Revised: January 5, 2013 